Check for updates

Cite this: Soft Matter, 2017, 13,3480

Received 16th January 2017, Accepted 25th April 2017

DOI: $10.1039 / \mathrm{c} 7 \mathrm{sm} 00108 \mathrm{~h}$

rsc.li/soft-matter-journal

\section{The role of optical projection in the analysis of membrane fluctuations $\dagger$}

\author{
S. Alex Rautu, ${ }^{a}$ Davide Orsi, (D) ${ }^{\mathrm{b}}$ Lorenzo Di Michele, ${ }^{\mathrm{c}}$ George Rowlands, ${ }^{a}$ \\ Pietro Cicuta (D) ${ }^{\star c}$ and Matthew S. Turner ${ }^{\star a}$
}

The spectral analysis of thermal fluctuations, or flickering, is a simple and non-invasive method widely used to determine the mechanical properties of artificial and biological lipid membranes. In its most common implementation, the position of the edge of a cell or vesicle is tracked from optical microscopy videos. However, a systematic disagreement with X-ray scattering and micromechanical manipulation data has brought into question the validity of the method. We present an improved analysis protocol that resolves these discrepancies by accounting for the finite vertical resolution of the optics used to image fluctuations.

The spectroscopy of shape fluctuations is a general technique to infer the mechanical properties of soft and biological matter. Specifically, fluid membranes are very soft and analysis of shape conformations allows one to characterise physical properties and aspects of the biochemical activity: the "flickering" of giant unilamellar vesicles (GUVs) and red blood cells (RBC) is a classic example. ${ }^{1}$ In flicker spectroscopy, the equatorial fluctuations of GUVs are imaged through video microscopy and reconstructed by image analysis, yielding a power spectrum that is then compared to a theoretical model to retrieve the membrane tension $\sigma$ and the bending rigidity $\kappa$. The latter is an intrinsic property of the membrane that affects its dynamics and structure, as well as morphology, motility, and endocytosis in living cells. ${ }^{2}$ Flickering experiments are widely used to assess the differences in the

\footnotetext{
${ }^{a}$ Department of Physics, University of Warwick, Coventry, CV4 7AL, UK.

E-mail: m.s.turner@warwick.ac.uk; Tel: +44 (0)24 76522257

${ }^{b}$ Department of Mathematics, Physics and Computer Sciences, University of Parma, Parma, Italy

${ }^{c}$ Cavendish Laboratory, University of Cambridge, Cambridge, CB3 OHE, UK. E-mail: pc245@cam.ac.uk; Tel: +44 (0)1223 337462

$\dagger$ Electronic supplementary information (ESI) available: We provide details on the preparation of GUVs, on the confocal microscopy setup, on the code used to extract the fluctuation spectrum from a video of a GUV, and on the data analysis procedure. We report the complete theoretical derivation of the model at the basis of our Method. We also provide the Matlab routine used to analyse fluctuations of GUVs with example and the Mathematica routine used to fit the fluctuation spectrum and retrieve the mechanical properties of the membrane. See DOI: $10.1039 / \mathrm{c} 7 \mathrm{sm} 00108 \mathrm{~h}$
}

Table 1 Measurements of the bending rigidity $\kappa$ (in units of $k_{\mathrm{B}} T$ ) for DOPC lipid membranes from different experimental techniques, including the estimate of the present work and the previous literature values (shown as a weighted average of the estimates from each cited work)

\begin{tabular}{ll}
\hline Experimental method & $\kappa / k_{\mathrm{B}} T$ \\
\hline Flicker spectroscopy of GUVs & \\
Literature values $^{10,11}$ & $28 \pm 3$ \\
Present work - no optical projection & $27 \pm 1$ \\
Present work - optical projection & $19 \pm 1$ \\
Active driving of GUVs & $21 \pm 3$ \\
Pulling membrane tethers $^{12,13}$ & $20 \pm 4$ \\
X-ray scattering on bilayer stacks $^{14-17}$ & $18 \pm 2$ \\
Micropipette aspiration of GUVs $^{18-20}$ & $20 \pm 2$
\end{tabular}

membrane rigidity of various lipid compositions, since only a basic microscope is needed, the sample preparation procedures are well established and direct manipulation of the membrane can be avoided. However, the absolute value of $\kappa$ measured via flickering is systematically larger than those obtained by X-ray scattering, direct manipulation of GUVs and active driving, ${ }^{3}$ as reported in Table 1 for 1,2-dioleoyl-sn-glycero-3-phosphocholine (DOPC). Here we show that this discrepancy arises from the inadequacy of the theoretical models currently used to fit experimental data. Specifically, these ignore the finite focal depth of the setups used to image the membranes, which ultimately projects some out-of-focus fluctuations onto the imaging plane. We introduce a theoretical model that accurately accounts for these projections, and test it on experimental data collected with a fast scanning confocal microscope, in which the focal depth is precisely set. ${ }^{4}$ When compared with standard flickering analysis on DOPC vesicles (that gives $\kappa=28 \pm 3 k_{\mathrm{B}} T$ ), we observe a $30 \%$ reduction in the measured values of $\kappa$ and find an excellent agreement with the alternative experimental methods. Our technique can be applied to any optical microscopy setup, once the focal depth is known; a proof-of-concept analysis of a phase contrast imaging experiment is reported in the ESI. $\dagger$

A fluctuating GUV can be described as a quasi-spherical shell $S \equiv R[1+u(\theta, \varphi)] \hat{\mathbf{r}}$, where $(\theta, \varphi)$ are the spherical angular coordinates, $u(\theta, \varphi)$ is a small deviation about a sphere of radius 
(a)

(c)

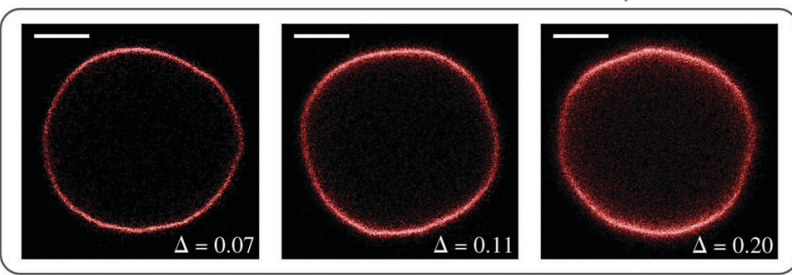

Fig. 1 (a) Schematic diagram of a fluctuating vesicle. Here, the focal plane of the microscope is shown in light blue, while the green slab depicts the region within the focal depth $\Delta$ (hereinafter, non-dimensionalised by the mean vesicle radius), where the surface modes are averaged in projection. Here, $\varphi \in[0,2 \pi)$ measures the azimuthal angle, while $\theta \in[0, \pi]$ measures the zenith angle or latitude. (b) Sketch of a scalar field $\mathscr{I}(r, \varphi)$, corresponding to the membrane density (mass, or intensity for fluorescent GUVs) projected onto the focal plane from within the focal depth (red is high density, blue is low). The solid black line represents the first radial moment of $\mathscr{I}(r, \varphi)$, written $\rho_{\Delta}(\varphi)$. (c) Confocal fluorescence images of a GUV of mean radius $R=10.2 \mu \mathrm{m}$ at increasing values of $\Delta$, as fluorescence is captured from a greater area of membrane. The radial distribution reflects the underlying membrane flickering. The scale bar is $5 \mu \mathrm{m}$.

$R$, and $\hat{\mathbf{r}}$ is the radial unit vector (see Fig. 1a). The free-energy of the vesicles is described by the Helfrich Hamiltonian, which depends on $\kappa$ and $\sigma .^{5}$ By expanding this Hamiltonian to second order in $u$, written in the basis of the spherical harmonics $Y_{n}^{m}$ as $u(\theta, \varphi)=\sum_{n=0} \sum_{|m| \leq n} \mathscr{U}_{n}^{m} Y_{n}^{m}(\theta, \varphi)$, with $\mathscr{U}_{n}^{m}$ the mode amplitude, one can express the time-averaged mean square fluctuation amplitudes as follows: ${ }^{6}$

$$
\left\langle\left|\mathscr{U}_{n}^{m}\right|^{2}\right\rangle=\frac{k_{\mathrm{B}} T}{\kappa(n-1)(n+2)[n(n+1)+\bar{\sigma}]},
$$

where $n$ and $m$ are the integer mode numbers with $n \geq 2$ and $|m| \leq n, \bar{\sigma}=\sigma R^{2} / \kappa-2 H_{0} R+2 H_{0}{ }^{2} R^{2}$ is the reduced tension, $H_{0}$ is the mean spontaneous curvature, $k_{\mathrm{B}}$ is the Boltzmann constant, and $T$ is the temperature. The decay time of each mode is given by: ${ }^{6}$

$$
\tau_{n}=\frac{R^{3}\left[\eta_{\text {in }} \frac{(n+2)(2 n-1)}{n+1}+\eta_{\text {out }} \frac{(n-1)(2 n+3)}{n}\right]}{\kappa(n-1)(n+2)[n(n+1)+\bar{\sigma}]},
$$

with $\eta_{\text {in }}$ and $\eta_{\text {out }}$ as the viscosities of the fluid found inside and outside of the vesicle, respectively.

Membrane fluctuations are recorded by microscopy imaging in $2 \mathrm{D}$, normally around the equatorial plane. Thus, experimental data have been typically compared with mode amplitudes obtained by projecting the modes in eqn (1) onto the plane $\theta=\pi / 2$; see ref. 7 and the ESI $\dagger$ for a full derivation. However, due to finite depth of focus, the experiments cannot isolate the signal from the equatorial plane alone. What is actually observed is a projection over the strip of membrane that lies within a focal region near the equator, as shown in Fig. 1(a); this strip can support a spectrum of surface modes that are partially averaged out in projection. This averaging effect is expected to be particularly strong for modes $q \gtrsim 1 / \Delta$, where $\Delta$ is a non-dimensional parameter given by the ratio of the focal depth of the microscope to the vesicle radius $R$.

In the case of fluorescence microscopy, where the membrane is assumed to be uniformly labelled with fluorophores that emit isotropically (see Fig. 1b), we idealise the acquired optical signal as a convolution of the membrane shape with a Gaussian of width equal to the focal depth. Namely, light arriving from height $z$ above (or below) the focal plane has intensity scaled by $\mathscr{G}(z)=\exp \left[-\frac{1}{2} z^{2}(R \Delta)^{-2}\right]$. Thus, the projected intensity field on the equatorial plane is

$$
\begin{aligned}
& \mathscr{I}(r, \varphi) \propto \int \mathrm{d} \Omega \mathscr{G}\left(r^{\prime} \cos \theta^{\prime}\right) \delta\left(r-r^{\prime} \sin \theta^{\prime}\right) \\
& \delta\left(r^{\prime} \varphi^{\prime} \sin \theta^{\prime}-r \varphi\right) \delta\left(r^{\prime}-R\left[1+u\left(\theta^{\prime}, \varphi^{\prime}\right)\right]\right),
\end{aligned}
$$

where $\int \mathrm{d} \Omega \equiv \int_{0}^{\infty} \mathrm{d} r^{\prime} r^{\prime 2} \int_{0}^{\pi} \mathrm{d} \theta^{\prime} \sin \theta^{\prime} \int_{0}^{2 \pi} \mathrm{d} \varphi^{\prime}, r$ and $\varphi$ are the polar coordinates, and $\delta$ is the Dirac delta function. Experiments detect $\mathscr{I}(r, \varphi)$ and locate the apparent membrane contour as the first radial moment $\rho_{\Delta}(\varphi)=\int_{0}^{\infty} r \mathscr{I}(r, \varphi) \mathrm{d} r / \int_{0}^{\infty} \mathscr{I}(r, \varphi) \mathrm{d} r$. Deviations of $\rho_{\Delta}(\varphi)$ are analyzed in Fourier space, where they are written as $\mu_{q}(t)$ and are non-dimensionalised by $R$. By defining $\bar{\mu}_{q}(t) \equiv \tau^{-1} \int_{0}^{\tau} \mathrm{d} t^{\prime} \mu_{q}\left(t+t^{\prime}\right)$ to account for microscope exposure time $\tau$, the mean square amplitude can be exactly computed

$$
\left\langle\bar{\mu}_{q}(t) \bar{\mu}_{q}^{*}(t)\right\rangle=\sum_{n \geq q} \mathscr{L}_{n, q}{ }^{2}\left\langle\left|\mathscr{U}_{n}^{q}\right|^{2}\right\rangle \frac{\tau_{n}^{2}}{\tau^{2}}\left[1-\mathrm{e}^{-\frac{\tau}{\tau_{n}}}\right]^{2},
$$

where $\mathscr{L}_{n, q}=\int_{0}^{1} \mathrm{~d} \omega \mathscr{P}_{n}^{q}(\omega) f_{\Delta}(\omega)\left[1+(-1)^{n+q}\right]$, with $\mathscr{P}_{n}^{q}(\cos \theta) \equiv Y_{n}^{q}$ $(\theta, \varphi=0)$, and $f_{\Delta}(\omega)$ is defined by

$$
f_{\Delta}=\frac{\frac{\omega^{2}+2 \Delta^{2}}{\Delta^{2}} \mathrm{e}^{\frac{1-2 \omega^{2}}{4 \Delta^{2}}}}{\pi I_{0}\left[\frac{1}{4 \Delta^{2}}\right]}-\frac{\frac{\omega^{2}+\Delta^{2}}{\Delta \sqrt{1-\omega^{2}}} \operatorname{erf}\left[\frac{1}{\Delta \sqrt{2}}\right] \mathrm{e}^{\frac{1-\omega^{2}}{2 \Delta^{2}}}}{\pi I_{0}^{2}\left[\frac{1}{4 \Delta^{2}}\right] \sqrt{\frac{\pi}{2}}},
$$

where erf is the error function, and $I_{0}$ is the modified Bessel function of the first kind of order zero. ${ }^{8}$ A complete derivation of eqn (4) and (5), and the comparison with the standard formula (which is recovered in the limit $\Delta \rightarrow 0$ ) is provided in the ESI. $\dagger$

The experiments are performed on GUVs prepared by means of electroformation as in ref. 9, with DOPC and the fluorescent lipid Texas Red 1,2-dihexadecanoyl-sn-glycero-3-phosphoethanolamine (DHPE) in proportions of $99.2 \%$ and $0.8 \%$, respectively. Videos of the equatorial fluctuations of GUVs are collected on a Leica TCS SP5 II scanning confocal microscope, using a HCX-PLAPO-CS 40.0 $\times$ oil immersion objective with numerical aperture $\mathrm{NA}=1.30$. The focal depth is defined by the pinhole size of the microscope, which allows us to experimentally obtain the value of $\Delta$ by fitting the intensity profile along the $z$-direction to a Gaussian form of standard deviation $R \Delta$. The position of the equatorial contours in every frame is determined with sub-pixel precision by correlating the radial intensity profile with a template. 
By Fourier transforming the contours and averaging over $\sim 2000$ frames, we extract the mean-squared amplitude of the equatorial modes $F_{q}(\Delta)$, which can be directly compared with the model (eqn (4)); see ESI $\dagger$ for full experimental details. Note that a cutoff condition in the mode spectrum, $q \lesssim Q$, arises due the finite temporal resolution of the microscope, particularly the rate at which the illumination spot is raster-scanned across the field of view. With our setup we typically obtain $Q \approx 20$ (see ESI $\dagger$ ). The accessible wave number is also limited by the spatial resolution of the setup to $q \lesssim q_{\mathrm{w}}=R / \mathscr{W}$, where $\mathscr{W}$ is the lateral width of the diffraction-limited illumination spot.

Using eqn (4), the best-fit values of $\kappa$ and $\bar{\sigma}$ to the experimental spectrum $F_{q}(\Delta)$ are found by means of a maximum posterior estimate, ${ }^{21}$ assuming a uniform prior and that the measurement errors are independent and Gaussian; namely, we seek to minimise

$$
\chi_{\Delta}{ }^{2}(\kappa, \bar{\sigma})=\sum_{q=q_{\min }}^{q_{\max }} \frac{\left[\left\langle\bar{\mu}_{q}(t) \bar{\mu}_{q}^{*}(t)\right\rangle-F_{q}(\Delta)\right]^{2}}{\Sigma_{q}^{2}(\Delta)},
$$

where $\Sigma_{q}(\Delta)$ is the standard error in the mean associated with $F_{q}(\Delta)$. Here, $q_{\mathrm{min}}$ and $q_{\mathrm{max}}$ define the lower and upper bounds of the fitting range, respectively, with the former chosen to be $q_{\min }=3$. Due to the rapid convergence to zero of $\mathscr{L}_{n, q}{ }^{2}$, the sum in eqn (4) is truncated at the mode $n=q+30$. On the other hand, the upper bound of the fitting range is selected as one that maximises the posterior probability $\mathbb{P}\left(q_{\text {max }} \mid D_{\Delta}\right)$ based on data $D_{\Delta}=\left\{F_{q}(\Delta)\right\}_{q}$. This can be numerically computed, but further analytical progress can be made by expanding $\chi_{\Delta}{ }^{2}(\kappa, \bar{\sigma})$ to second order around the best-fit values of $\kappa$ and $\bar{\sigma}$, which yields the following expression:

$$
\mathbb{P}\left(q_{\max } \mid D_{\Delta}\right) \propto \frac{\mathrm{e}^{-\chi_{\min }{ }^{2} / 2}}{\sqrt{\operatorname{det}\left(\mathbf{H}_{\min }\right)}} \prod_{q=q_{\min }}^{q_{\max }} \frac{1}{\Sigma_{q}(\Delta)},
$$

where $\chi_{\min }{ }^{2}=\min \left[\chi_{\Delta}{ }^{2}(\kappa, \bar{\sigma})\right]$, and $\mathbf{H}_{\min }$ is the Hessian matrix of eqn (6) evaluated at the best-fit values. We also impose that $q_{\max }$ must be greater than the crossover $q_{\mathrm{c}} \equiv R \sqrt{\sigma / \kappa}$, $\neq$ and less than the cutoffs $q_{\mathrm{w}}$ and $Q$. In other words, the optimal fit is achieved when $\chi_{\Delta}{ }^{2}(\kappa, \bar{\sigma})$ is minimal and simultaneously its upper bound $q_{\max } \in\left(q_{\mathrm{c}}, \min \left(q_{\mathrm{w}}, Q\right)\right]$ maximises the probability in eqn (7). If $q_{\text {max }}$ lies outside the interval discussed above, then the dataset $D_{\Delta}$ is rejected. See ESI $\dagger$ for details, code and example files. The maximum posterior estimate can be applied to datasets $\mathscr{D}$ containing spectra from $N$ different vesicles imaged with different $\Delta$. In this case, since $\sigma$ is not a material property of the bilayer, the posterior probability becomes an $(N+1)$-dimensional function $\mathbb{P}\left(\kappa, \bar{\sigma}_{1}, \ldots \bar{\sigma}_{N} \mid \mathscr{D}\right)$, with a different $\bar{\sigma}_{\ell}$ for each $\ell$-th vesicle. As in eqn (6), we assume a uniform prior and that the measurement errors are independent and Gaussian; thus, maximising this posterior probability function is equivalent to minimizing $\chi_{\mathscr{D}}^{2} \equiv$ $\Sigma_{\Delta} \Sigma_{\ell} \chi_{\Delta}^{2}\left(\kappa, \bar{\sigma}_{\ell}\right)$, where each individual $q_{\max }$ is given by the maximum of eqn (7).

By imaging three GUVs with radii between 6-17 $\mu \mathrm{m}$ at various pinhole sizes, the fluctuation spectrum associated with each $\Delta$ yields an individual estimate for $\kappa$ and $\sigma$. A systematic decrease in the inferred value of $\kappa$ is found when the data is fitted with the model in eqn (4) in comparison with the "standard" model that considers only equatorial fluctuations, i.e. $\Delta=0$ (see Fig. 2a). Using a maximum posterior estimate based on the data of all the vesicles imaged at different $\Delta$, we find $\kappa=19 \pm 1 k_{\mathrm{B}} T$ (Fig. 2b), in perfect agreement with literature values obtained with $\mathrm{X}$-rays and micromanipulation techniques (see Table 1). In contrast, by fitting all spectra with the conventional model $(\Delta=0)$, we find that $\kappa=27 \pm 1 k_{\mathrm{B}} T$, a value compatible with the literature estimates of $\kappa$ obtained by conventional flickering analysis (see Table 1).

Large discrepancies in the inferred surface tension values are also to be expected. To illustrate the dependence of the inferred values of $\kappa$ with the focal depth, the conventional fitting procedure is repeated at arbitrary non-zero values of $\Delta$ for all of the spectra in $\mathscr{D}$, yielding an interpolated curve depicted by the green line in Fig. 2b. This shows how increasing the focal depth can result in an apparent increase in $\kappa$ if its effects are not properly analysed. (a)

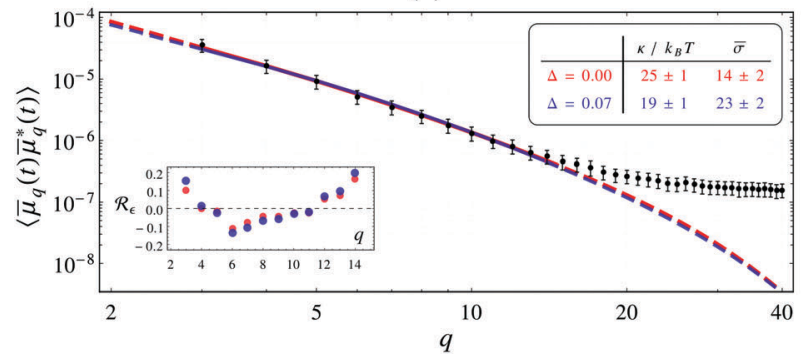

(b)

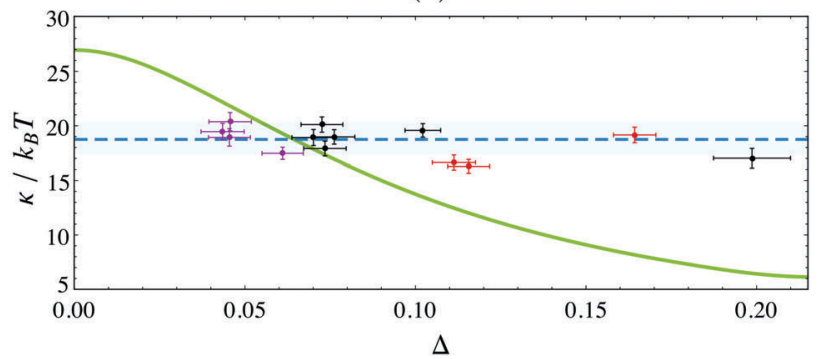

Fig. 2 (a) Fluctuation spectrum for a GUV $(R \approx 10.2 \mu \mathrm{m})$ imaged via confocal fluorescence microscopy, with $\Delta=0.07$ and $\tau \approx 1.2 \mathrm{~ms}$, on a log-log scale. Lines are the best-fit for the "standard" model (i.e. incorrectly assuming $\Delta=0$, red), and eqn (4) with $\Delta=0.07$ (blue). Both fits are of similar quality, but their best-fit values are significantly different, see the inset table (same colors). Dashed lines give their extrapolation outside the fitting range. The errorbars are standard errors in the mean, scaled up by a factor 10 to improve visibility. Inset plot shows the residuals $R_{\varepsilon}(q)$, normalised to the standard deviation. (b) Values of $\kappa$ from the analysis of three GUVs of radii $6.4 \mu \mathrm{m}$ (purple), $10.2 \mu \mathrm{m}$ (black), and $16.7 \mu \mathrm{m}$ (red), observed at different values of $\Delta$. The blue dashed line is the estimate of $\kappa$ from the entire dataset $\mathscr{D}$, found by maximizing the posterior probability $\mathbb{P}\left(\kappa, \bar{\sigma}_{1}, \bar{\sigma}_{2}, \bar{\sigma}_{3} \mid \mathscr{D}\right)$, where $\bar{\sigma}_{\ell}$ is the corresponding reduced surface tension of $\ell$-th vesicle. This yields $\kappa=19 \pm 1 k_{\mathrm{B}} T$ (the blue band is the $95 \%$ confidence interval). The green curve shows $\kappa$ inferred by fitting the data $\mathscr{D}$ at fixed $\Delta$. Large errors arise if $\Delta$ is not accounted for correctly; the value $\kappa \approx 27 k_{\mathrm{B}} T$, found at $\Delta=0$, corresponds to the use of the "standard" model to fit the experiments. The correction is also important for GUVs of varying radius, even when imaged with same optics. 
In conclusion, a new methodology is developed for the analysis of flickering data that can accurately account for the finite focal depth of the optical imaging setups. Thus, this resolves a systematic inconsistency in the estimated values of bending rigidity, bringing flickering into full quantitative agreement with other methods such as X-ray scattering and micromanipulation techniques. Neglecting this correction leads to a systematic and size-dependent error which is unfortunately present in all (hundreds) published papers. Using our approach, flickering analysis is now competitive in terms of accuracy with the aforementioned methods, allowing the user to exploit its several advantages in terms of easiness of use and simplicity, as it relies on general purpose and easily accessible equipment, it is noninvasive, and can be integrated into microfluidic devices.

\section{Acknowledgements}

We acknowledge stimulating discussions with M. Rao, P. Sens, and A. T. Brown, the sample preparation support from L. Parolini, and the help on simulations on the scanning sampling from A. Javer. This research work is funded by EPSRC under grants EP/I005439/1 (Matthew Turner) and EP/J017566/1 (Pietro Cicuta), Project SPINNER 2013, Regione Emilia-Romagna, European Social Fund (Davide Orsi), The Leverhulme Trust and the Isaac Newton Trust through an Early Career Fellowship, the Ernest Oppenheimer Fund, and Emmanuel College Cambridge (Lorenzo Di Michele). A complete dataset in support of this publication is available free of charge at https://doi.org/10.17863/CAM.9209.

\section{References}

\# This crossover $q$-mode separates the regimes in which the membrane is dominated by the surface tension term $\left(q \lesssim q_{\mathrm{c}}\right)$ and the bending rigidity term $\left(q \gtrsim q_{\mathrm{c}}\right)$. Thus, we require its value to lie within the fitting range.

1 P. Bassereau, B. Sorre and A. Lévy, Adv. Colloid Interface Sci., 2014, 208, 47-57.

2 R. Lipowsky and E. Sackmann, Structure and Dynamics of Membranes: I. From Cells to Vesicles, Elsevier Science, Amsterdam, 1995.
3 J. F. Nagle, M. S. Jablin, S. Tristram-Nagle and K. Akabori, Chem. Phys. Lipids, 2015, 185, 3-10.

$4 \mathrm{~J}$. Mertz, Introduction to Optical Microscopy, Roberts and Company Publishers, Greenwood Village, 2009.

5 U. Seifert, Adv. Phys., 1997, 46, 13-137.

6 S. T. Milner and S. A. Safran, Phys. Rev. A: At., Mol., Opt. Phys., 1987, 36, 4371-4379.

7 J. Pécréaux, H. G. Döbereiner, J. Prost, J.-F. Joanny and P. Bassereau, Eur. Phys. J. E: Soft Matter Biol. Phys., 2004, 13, 277-290.

8 M. Abramowitz and I. Stegun, Handbook of Mathematical Functions, Dover Publications Inc., New York, 1965.

9 L. Parolini, B. M. Mognetti, J. Kotar, E. Eiser, P. Cicuta and L. Di Michele, Nat. Commun., 2015, 6, 5948.

10 A. T. Brown, J. Kotar and P. Cicuta, Phys. Rev. E: Stat., Nonlinear, Soft Matter Phys., 2011, 84, 021930.

11 R. S. Gracià, N. Bezlyepkina, R. L. Knorr, R. Lipowsky and R. Dimova, Soft Matter, 2010, 6, 1472.

12 A. Tian, B. R. Capraro, C. Esposito and T. Baumgart, Biophys. J., 2009, 97, 1636-1646.

13 B. Sorre, A. Callan-Jones, J.-B. Manneville, P. Nassoy, J.-F. Joanny, J. Prost, B. Goud and P. Bassereau, Proc. Natl. Acad. Sci. U. S. A., 2009, 106, 5622-5626.

14 N. Kucerka, Y. Liu, N. Chu, H. I. Petrache, S. Tristram-Nagle and J. F. Nagle, Biophys. J., 2005, 88, 2626-2637.

15 N. Kucerka, S. Tristram-Nagle and J. F. Nagle, J. Membr. Biol., 2006, 208, 193-202.

16 J. Pan, T. T. Mills, S. Tristram-Nagle and J. F. Nagle, Phys. Rev. Lett., 2008, 100, 198103.

17 J. Pan, S. Tristram-Nagle, N. Kucerka and J. F. Nagle, Biophys. J., 2008, 94, 117-124.

18 W. Rawicz, K. C. Olbrich, T. McIntosh, D. Needham and E. Evans, Biophys. J., 2000, 79, 328-339.

19 N. Fa, L. Lins, P. J. Courtoy, Y. Dufrêne, P. Van Der Smissen, R. Brasseur, D. Tyteca and M.-P. Mingeot-Leclercq, Biochim. Biophys. Acta, 2007, 1768, 1830-1838.

20 P. Shchelokovskyy, S. Tristram-Nagle and R. Dimova, New J. Phys., 2011, 13, 25004.

21 D. S. Sivia and J. Skilling, Data Analysis - A Bayesian tutorial, Oxford University Press, Oxford, 2nd edn, 2006. 